\title{
Orientation isotope effect in ion-molecule reactions
}

\author{
Peter M. Hierl \\ Department of Chemistry, University of Kansas, Lawrence, Kansas 66045 \\ (Received 14 May 1976; revised paper received 17 February 1977)

\begin{abstract}
A simple kinematic model is proposed to account for the kinetic isotope effect (KIE) upon the reactions of simple ions $\mathrm{X}^{+}$with HD. This model is based upon (1) the fact that the displacement of the center of polarizability from the center of mass in the HD molecule will affect the alignment of the reactants, (2) the fact that, for many ions $\mathrm{X}^{+}$, reaction must occur by a surface crossing mechanism, and (3) the assumption that the ratio $\mathrm{XH}^{+} /\left(\mathrm{XH}^{+}+\mathrm{XD}^{+}\right)$equals the fraction of intimate collisions in which the $\mathrm{H}$ end of $\mathrm{HD}$ is oriented towards the ion at the moment the reactants pass over the centrifugal barrier in the effective radial potential (or cross over to the $\mathrm{X}-\mathrm{HD}^{+}$surface if the crossing occurs before the centrifugal barrier is reached). Use of the ion-induced dipole potential for the reactants permits the derivation of an analytic expression for the KIE. With no adjustable parameters, this model accounts quantitatively for the very different $\mathrm{KIE}$ 's observed in the reactions of $\mathrm{Ar}^{+}$and $\mathrm{Kr}^{+}$with $\mathrm{HD}$ at low collision energies.
\end{abstract}

\section{INTRODUCTION}

As reported previously, ${ }^{1,2}$ the intramolecular kinetic isotope effect (KIE) upon the ion-molecule reaction

$$
\begin{aligned}
\mathrm{Kr}^{+}+\mathrm{HD} & \rightarrow \mathrm{KrH}^{+}+\mathrm{D} \\
& \rightarrow \mathrm{KrD}^{+}+\mathrm{H}
\end{aligned}
$$

displays a very unusual energy dependence. The ratio $\sigma\left(\mathrm{KrH}^{+}\right) / \sigma\left(\mathrm{KrD}^{+}\right)$increases rapidly with collision energy, passes through a maximum of about 2.5 at $0.7 \mathrm{eV}$ relative energy, and then decreases to a value of about 0.3 at collision energies above $3 \mathrm{eV}$. Both the sharp maximum at low energies $(E<1.5 \mathrm{eV})$ and the inverse isotope effect at high energies $(E>1.5 \mathrm{eV})$ are quite extraordinary, being in marked contrast with the results reported by other workers for the analogous reactions of $\mathrm{Ar}^{+}, \mathrm{N}_{2}^{+}$, and other simple ions with HD. ${ }^{3-6}$ In these cases the KIE was near unity at the lowest collision energy and increased (slowly at first but then more rapidly) with increasing collision energy, reaching values greater than 2 only at rather high $(>4 \mathrm{eV})$ collision energies. Theoretical models based upon complex formation, ${ }^{3}$ energy and momentum conservation, ${ }^{5}$ potential energy surfaces, ${ }^{7-9}$ and various kinematic models ${ }^{10-14}$ have been proposed to explain the isotope effects observed in these earlier studies. None of these models, however, seem adequate to explain the unusual KIE displayed by Reaction (1).

We propose here a simple reaction model which accounts quite well for the KIE observed in Reaction (1) at collision energies less than $1.5 \mathrm{eV}$ center of mass (c. m. ). This model, which contains no adjustable parameters, has as its physical basis two facts which are common to a number of ion-molecule reactions involving $\mathrm{HD}$.

First, in a molecule such as $\mathrm{HD}$, the center of mass is displaced from the center of polarizability by an amount $\Delta r$ equal to one-sixth the HD separation. In an ion-molecule reaction of the general type

$$
\begin{aligned}
\mathrm{X}^{+}+\mathrm{HD} & \rightarrow \mathrm{XH}^{+}+\mathrm{D} \\
& \rightarrow \mathrm{XD}^{+}+\mathrm{H},
\end{aligned}
$$

this displacement will cause the intermolecular potential governing the collision of the reactants to depend upon both the distance $r$ from $\mathrm{X}^{+}$to the center of mass of $\mathrm{HD}$ and the angle $\theta$ between the HD bond axis and the vector $r$ (see Fig. 1). As a result of the anisotropy in the potential, certain orientations of the reactants will be more probable than others. If one assumes the $\mathrm{xH}^{+}$is formed when the $\mathrm{H}$ end of the $\mathrm{HD}$ is directed toward the ion, the ratio $\sigma\left(\mathrm{XH}^{*}\right) / \sigma\left(\mathrm{XD}^{+}\right)$can be calculated if one knows the probability of a particular orientation, $P(\theta)$, as a function of the relative collision energy $E$.

Second, for many ion-molecule reactions of the type $\mathrm{X}^{+}+\mathrm{H}_{2}-\mathrm{XH}^{+}+\mathrm{H}$, the ground state of $\mathrm{XH}^{+}$dissociates to $\mathrm{X}+\mathrm{H}^{+}$, so that only the reactant state $\mathrm{X}+\mathrm{H}_{2}^{+}$correlates with the ground state of the product. Therefore, these reactions must occur by a surface crossing mechanism from the initial $\mathrm{X}^{+}-\mathrm{H}_{2}$ surface to the $\mathrm{X}-\mathrm{H}_{2}^{+}$surface. The position of this crossing, which is strongly influenced by the ionization potential of $\mathrm{X}$, can be expected to have a marked effect on the collision dynamics. ${ }^{15}$

George and Suplinskas, ${ }^{14}$ using the "kinematic model for reaction" to calculate the KIE in the reaction of $\mathrm{Ar}^{+}$ with $\mathrm{HD}$, have previously considered the effect of molecular reorientation upon the product yield. At collision energies above $2 \mathrm{eV}$ (c. m.), the predictions of their model were in agreement with experimental results. At these energies, however, the predicted KIE was determined principally by the problem of energy disposal; the orientation effect produced only a small dif-

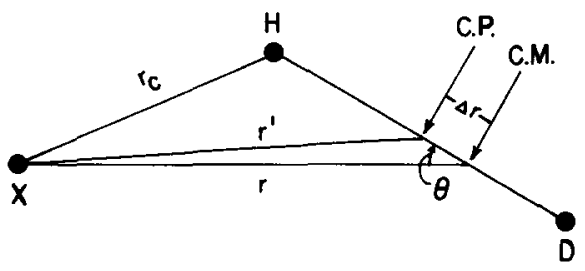

FIG. 1. Schematic representation of the $\mathrm{X}^{+}-\mathrm{HD}$ system, defining the coordinates discussed in the text. The force exerted upon the HD molecule is assumed to be directed towards the center of polarizability, which is taken as the geometric center of the molecule and is marked C. P. The HD molecule rotates about its center of mass (c. m.), displaced a distance $\Delta r$ from the C.P. The distance from $\mathrm{X}^{+}$to the center of polarizability is $r^{\prime}$. The angle $\theta$ defines the orientation of the $\mathrm{H}$ end of $\mathrm{HD}$ with respect to $r$. 
ference in the calculated results. Although the orientation effect was found to have a greater effect upon the KIE at lower collision energies, the kinetic model failed to account quantitatively for the observed KIE at collision energies below $2 \mathrm{eV}$. The authors attributed this failure of the model to uncertainties in the reaction exoergicity and the intermolecular potentials.

The model proposed here considers only the effect of reactant orientation upon the product ratio and ignores other effects (kinematic considerations, statistical effects, etc.) which may influence the ratio of the isotropic products. In Sec. II we present the details of the model used to calculate the orientation isotope effect. The application of this model to the reactions of $\mathrm{Kr}^{+}$and of $\mathrm{Ar}^{+}$with HD is discussed in Sec. III.

\section{DESCRIPTION OF THE MODEL}

\section{A. Intermolecular potential}

Quantitative application of this model requires an analytic expression for the intermolecular potential between the reactants $\mathrm{X}^{+}-\mathrm{H}_{2}$. Because such potentials, particularly at small distances of separation, are only imperfectly understood at this time, it has been customary to approximate the interaction between an ion and a polarizable molecule by the ion-induced dipole potential. Although the inadequacy of this approximation is now generally recognized, ${ }^{16}$ this approach has in the past provided useful insights to ion-neutral interactions. Moreover, because of its long-range nature, this potential can be expected to play a significant role in determining the orientation of the approaching reactants. Consequently, we choose to illustrate the features of this model by using this mathematically tractable expression for the potential:

Assumption 1. For the interaction of an ion with a polarizable molecule, the intermolecular potential is given by the ion-induced dipole potential

$$
V(r)=\left\{\begin{array}{ll}
-e^{2} \alpha / 2 r^{\prime 4} & \text { if } r>r_{e} \\
\infty & \text { if } r<r_{e}
\end{array},\right.
$$

where $e$ is the electronic charge, $\alpha$ the polarizability of the neutral species, and $r_{e}$ the hard sphere radius. The distance $r^{\prime}$ from the ion to the center of polarizability of $\mathrm{HD}$ is related to the distance $r$ from the ion to the center of mass of $\mathrm{HD}$ by the law of cosines (see Fig. 1):

$$
r^{\prime}=r\left(1-\frac{2 \Delta r \cos \theta}{r}+\frac{\Delta r^{2}}{r^{2}}\right)^{1 / 2} \text {. }
$$

Substitution of Eq. (4) into Eq. (3), and use of the approximation $(1-x)^{-n} \simeq 1+n x$ when $x \ll 1$, yields the intermolecular potential as a function of $r$ :

$$
V(r)=-e^{2} \alpha^{\prime} / 2 r^{4}-\Delta V \cos \theta,
$$

where

$$
\alpha^{\prime}=\alpha\left(1+2 \Delta r^{2} / r^{2}\right)
$$

and

$$
\Delta V=2 e^{2} \alpha \Delta r / r^{5} .
$$

In a collision at relative energy $E$ and impact parame- ter $b$, the effective radial potential is given by

$$
V_{\text {eff }}(r)=E b^{2} / r^{2}+V(r),
$$

where $V(r)$ is given by Eq. (5). The centrifugal force will cause the effective potential to have a maximum at $r_{\mathrm{max}}(E, b)$ of height $V_{\max }(E, b)$ :

$$
r_{\max }(E, b)= \begin{cases}\frac{1}{b}\left(\frac{2 e^{2} \alpha^{\prime}}{E}\right)^{1 / 2} & E<E_{c} \\ r_{8} & E \geqslant E_{c},\end{cases}
$$

where $E_{c}$, the energy at which the centrifugal barrier merges with the hard sphere repulsive wall, is given by $E_{c}=2 e^{2} \alpha^{\prime} / 2 \gamma_{B^{\circ}}^{4}$ The condition that $E$ must exceed the height $V_{\max }(E, b)$ for close collision to occur defines a maximum impact parameter for which close collisions are possible:

$$
b_{\max }(E)= \begin{cases}r_{e}\left(4 E_{c} / E\right)^{1 / 4} & E<E_{c} \\ \gamma_{e}\left(1+E_{c} / E\right)^{1 / 2} & E \geqslant E_{c} .\end{cases}
$$

The final relevant feature of the intermolecular potential applies to those reactions which proceed by a curve crossing mechanism. As shown by Gislason, ${ }^{17}$ the assumption of the ion-induced dipole potential leads to the conclusion that an $\mathrm{X}-\mathrm{H}_{2}^{+}$surface which lies $\Delta \epsilon$ above the initial $\mathrm{X}^{+}-\mathrm{H}_{2}$ surface at infinite separation will cross it at

$$
r_{x}=\left(e^{2} \Delta \alpha / 2 \Delta \epsilon\right)^{1 / 4} \text {, }
$$

where $\Delta \alpha=\alpha(\mathrm{X})-\alpha\left(\mathrm{H}_{2}\right)$. The crossings are presumably completely avoided in low energy collisions, so that $\mathrm{X}^{4}-\mathrm{H}_{2}$ goes adiabatically to $\mathrm{X}-\mathrm{H}_{2}^{+}$inside $r_{x} \cdot{ }^{17}$

\section{B. Evaluation of $\Delta V$}

The extent of molecular orientation depends upon the magnitude of $\Delta V$, which in turn varies as $r^{-5}$. Evaluation of the orientation isotope effect therefore requires the selection of a critical distance of separation, $r_{0}$, at which point the molecular orientation will determine the outcome of the collision. Two situations must be considered:

Case I: $\quad r_{\max }>r_{x}$. The reactants reach the maximum in the effective potential as $\mathrm{X}^{+}-\mathrm{H}_{2}$. Since their radial velocity will have its minimum value at $r_{\max }$, the reactants will have the opportunity for reorientation as they approach to this distance; once over the centrifugal barrier, the radial velocity will increase and collision will presumably occur soon enough that no further change in the molecular orientation can occur. In this case, therefore, we can identify $r_{0}$ with $r_{\text {max }}$. [Clearly, at very low collision energies $(E<0.1 \mathrm{eV}), r_{\max }$ is large enough and $\dot{r}$ small enough that sufficient time elapses after the barrier is surmounted for additional reorientation to occur. Application of this model, therefore, to such very low energy collisions is inappropriate.].

Case II: $r_{\max }<r_{x}$. Transition to the reactant state described by $\mathrm{X}-\mathrm{HD}^{+}$occurs before the distance $r_{\max }$ is achieved. Since the anisotropic potential given by Eq. (5) is no longer applicable inside $r_{x}$, we assume that no further molecular reorientation occurs beyond that already achieved during the approach to $r_{x}$. 
These considerations lead to the second (and key) assumption of this model:

Assumption 2. The ratio $\mathrm{XH}^{+} /\left(\mathrm{XH}^{+}+\mathrm{XD}^{+}\right)$is equal to the fraction of $\mathrm{HD}$ molecules with their $\mathrm{H}$ end oriented toward the ion (i. e., $-\pi / 2<\theta<\pi / 2$ ) at the moment the reactants pass over the centrifugal barrier in the effective potential or when they cross over to the $\mathrm{X}-\mathrm{HD}^{+}$surface, whichever occurs earlier in their approach. Thus, the critical value $r_{0}$ is given by

$$
r_{0}= \begin{cases}r_{\max } & r_{\max } \geqslant r_{x} \\ r_{x} & r_{\max }<r_{x}, .\end{cases}
$$

where $r_{x}$ is given by Eq. (11) and $r_{\max }$ is obtained by substituting Eq. (10) into Eq. (9):

$$
r_{\max }= \begin{cases}r_{e}\left(E_{c} / E\right)^{1 / 4} & E<E_{c} \\ r_{e} & E \geqslant E_{c} .\end{cases}
$$

The corresponding value of $\Delta V_{0}$ is then obtained by substituting into $\mathrm{Eq}$. (7) the appropriate value of $r_{0}$, as given in $\mathrm{Eq}$. (12).

\section{Orientation probability}

The angle-dependent portion of the potential,

$$
V_{\theta}=-\Delta V \cos \theta \text {, }
$$

will hinder the rotation of the HD molecule about its center of mass, thereby affecting the orientation probability, $P(\theta)$. For a given value of $\Delta V$, one can calculate the probability $P(\theta / J, \Delta V)$ of finding an HD molecule, whose rotational quantum number is $J$, at an orientation $\theta$.

\section{Low energy limit}

At very low collision energies, the collision time is so much longer than the rotational period of $\mathrm{HD}$ that the HD is able to orient itself under the influence of the angledependent portion of the intermolecular potential. Under these conditions, it can readily be shown ${ }^{18}$ that

$$
P(\theta / J, \Delta V) \propto \frac{\sin \theta}{\dot{\theta}} \propto \frac{\sin \theta}{\left(E_{J}+\Delta V \cos \theta\right)^{1 / 2}},
$$

where $E_{J}$ is the (thermal) rotational energy of HD in the plane of collision when the reactants are at infinite separation. For the sake of simplicity, in this paper we shall suppose that the HD reactant can rotate only in the collision plane. Under this restriction,

$$
E_{J}=J(J+1) B \text {, }
$$

where $B$ is the rotational constant of the HD molecule.

The fraction $F(J, E)$ of HD molecules in rotational state $J$ with $\theta$ between 0 and $\pi / 2$ (i.e., with the $H$ end closest to $\mathrm{X}^{+}$) at the moment the reactants reach the critical distance of separation, $r_{0}$, is given by

$$
F(J, E)=\frac{\int_{0}^{r / 2} P\left(\theta / J, \Delta V_{0}\right) d \theta}{\int_{0}^{\max } P\left(\theta / J, \Delta V_{0}\right) d \theta},
$$

where

$$
\theta_{\max }= \begin{cases}\pi & \text { if } E_{J} \geq \Delta V_{0} \\ \cos ^{-1}\left(-E_{J} / \Delta V_{0}\right) & \text { if } E_{J}<\Delta V_{0} .\end{cases}
$$

Integration of $P\left(\theta / J, \Delta V_{0}\right)$ yields an expression of the form

$$
\int P\left(\theta / J, \Delta V_{0}\right) d \theta=\frac{-2}{\Delta V_{0}}\left(E_{J}+\Delta V_{0} \cos \theta\right)^{1 / 2} .
$$

Evaluation at the appropriate limits of integration gives the following results:

Case I. $E_{J} \geq \Delta V_{0^{*}}$. The HD has sufficient energy to swing around in a complete circle, so that the motion is nonoscillating. With $\theta_{\max }=\pi$, Eq. (17) gives

$$
F(J, E)=\frac{\left(E_{J}+\Delta V_{0}\right)^{1 / 2}-\left(E_{J}\right)^{1 / 2}}{\left(E_{J}+\Delta V_{0}\right)^{1 / 2}-\left(E_{J}-\Delta V_{0}\right)^{1 / 2}} .
$$

Case II. $E_{J}<\Delta V_{0}$. The motion is oscillatory, with the maximum value of $\theta$ given by Eq. (18). Integration of Eq. (17) yields

$$
F(J, E)=\frac{\left(E_{J}+\Delta V_{0}\right)^{1 / 2}-\left(E_{J}\right)^{1 / 2}}{\left(E_{J}+\Delta V_{0}\right)^{1 / 2}} \quad \text { if } E_{J}<\Delta V_{0} \text {. }
$$

\section{High energy limit}

At very high collision energies, the collision occurs so rapidly (relative to the HD rotational period) that the HD has no time to become oriented in a manner consistent with the angle-dependent portion of the potential. In this energy region, $P(\theta / J, \Delta V)=\sin \theta$, and $F(J, E)$ $=0.5$.

In the range of intermediate energies, however, there will be a transition from the results of the preceding section to the high energy limit, $F(J, E)=0.5$. Physically, some HD molecules (those originally oriented roughly perpendicular to the approaching ion) will have time to rotate their $\mathrm{H}$ end toward the ion; other $\mathrm{HD}$ molecules (those with $\theta \sim \pi$ ) will not. Mathematically, this requires a weighting factor which describes the relative number of $\mathrm{HD}$ molecules capable of responding to potential $\Delta V$, as a function of the collision energy $E$. In order to have a simple analytic expression for this weighting factor, we make the following assumption:

Assumption 3. At a relative collision energy $E$, the fraction of HD molecules able to orient themselves appropriately during the time of interaction, $t_{c}$, is given by

$$
f(J, E)= \begin{cases}1 & \text { if } t_{c} \geq t_{r} \\ t_{c} / t_{r} & \text { if } t_{c}<t_{r}\end{cases}
$$

where $t_{r}$ is the time required for the HD molecule to rotate $\pi$ radians (i. e. , from the $\mathrm{X}^{*}-\mathrm{D}-\mathrm{H}$ to the $\mathrm{X}^{*}-\mathrm{H}-\mathrm{D}$ configuration). Of these molecules unable to orient themselves, $[1-f(J, E)]$, one-half are assumed to be oriented with $-\pi / 2<\theta<\pi / 2$. Therefore, the probability $R(J, E)$ that an HD molecule in a rotational state $J$ will be oriented with the $\mathrm{H}$ end towards $\mathrm{X}^{+}$in a collision at energy $E$ will be

$$
R(J, E)=f(J, E) F(J, E)+0.5[1-f(J, E)] .
$$

The interaction time $t_{c}$ is arbitrarily defined as

$$
t_{c}=\frac{r(1 \%)-r_{0}}{v_{r}}
$$

where $r(1 \%)$ is the $\mathrm{X}^{+}-\mathrm{HD}$ separation at which $\Delta V$ is only 


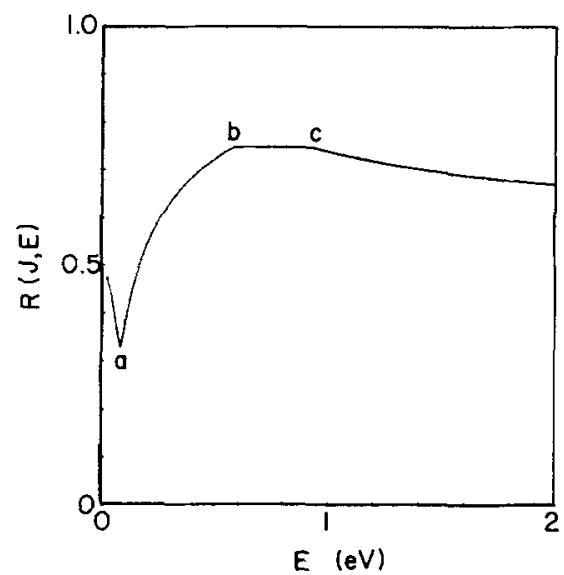

FIG, 2. Fraction of the HD molecules with their $\mathrm{H}$ end directed towards the ion $\mathrm{X}^{+}, R(J, E)$, as a function of the relative collision energy, $E$. This calculation was for the $\mathrm{Kr}^{+}-\mathrm{HD}$ system, with the HD in the $J=1$ rotational level. The causes of the changes in slope at points marked $a, b$, and $c$ are discussed in the text.

$1 \%$ of its maximum value, $\Delta V_{0}$ (which is achieved at separation $r_{0}$, and $v_{r}$ is the relative velocity. Since $\Delta V$ varies as $r^{-5}$, it is easily shown that

$$
r(1 \%)=(100)^{1 / 5} r_{0}=2.51 r_{0} \text {. }
$$

With the relative velocity taken as $(2 E / \mu)^{1 / 2}$, where $\mu$ is the reduced mass for translation of the reactants, Eq.

(24) becomes

$$
t_{c}=1.51 r_{0}(\mu / 2 E)^{1 / 2} .
$$

The average rotational velocity of $\mathrm{HD}$ is taken as $\left[2\left(E_{J}+\Delta V_{0}\right) / I\right]^{1 / 2}$, where $I$ is the moment of inertia of HD. The time required for HD to rotate $\pi$ radians is then $\pi$ divided by the average rotational velocity:

$$
t_{r}=\pi\left[I / 2\left(E_{J}+\Delta V_{0}\right)\right]^{1 / 2} \text {. }
$$

The probability $R(J, E)$ that HD in the $J=1$ level is oriented with its $H$ end towards the ion, as calculated from Eq. (23) using the potential for the $\mathrm{Kr}^{+}+\mathrm{HD}$ reaction, is illustrated in Fig. 2. The discontinuities in the slope of the function $R(J, E)$ have the following physical causes:

(a) $\Delta V\left(r_{0}\right)=E_{J}$. At this collision energy, $r_{0}$ has become sufficiently small [and $\Delta V\left(r_{0}\right)$ sufficiently large] that the transition occurs from $H D$ rotation at an uneven speed (which favors $\mathrm{XD}^{+}$formation) to partial locking in (or oscillation instead of rotation), which will increasingly favor $\mathrm{XH}^{+}$formation as $\Delta V_{0}$ increases further. That is, $\theta_{\max }$ becomes less than $\pi$ radians.

(b) The centrifugal barrier in the effective radial potential merges with the hard-sphere repulsive wall of the intermolecular potential, so that $r_{0}=r_{a}$ at this and higher energies. (In cases involving curve crossing, point $b$ would occur at the collision energy for which $r_{0}=r_{x}$.) Because $r_{0}$ remains constant as the collision energy is further increased, $\Delta V_{0}$ and consequently $R(J, E)$ remain constant.

(c) The collision energy has reached the high energy limit $\left(t_{c}=t_{r}\right)$, with the result that HD cannot react quickly enough to align itself in the energetically most favor- able orientation. As the collision energy is further increased (decreasing $t_{c}$ ) a decreasing fraction of the HD molecules will be able to complete their rotation during the interaction time, and $R(J, E)$ approaches its high-energy limit of 0.5 (i. e., totally random orientation).

Calculation of $R(J, E)$ for the $\mathrm{Kr}^{+}-\mathrm{HD}$ reaction (discussed in more detail in the following section) predicts that the various rotational states will exhibit dramatically different kinetic isotope effects (see Fig. 3). All HD molecules in the $J=0$ state, for example, become "locked in" to the $\mathrm{X}^{+}-\mathrm{H}-\mathrm{D}$ configuration at low collision energies $(E<0.5 \mathrm{eV})$, but are oriented in an increasingly random manner as the collision energy is increased beyond that for which $t_{c}$ equals $t_{r}$. HD molecules in the $J=1$ and 2 states, on the other hand, lead predominantly to $\mathrm{XD}^{+}$formation at the lowest collision energies (because the HD molecules are rotating more slowly when the $\mathrm{D}$ end is closest to the ion), and to $\mathrm{XH}^{+}$formation at collision energies above a few tenths of an $\mathrm{eV}$ (because the greater magnitude of $\Delta V_{0}$ at these higher energies causes a majority of the target molecules to become aligned in the $\mathrm{X}^{+}-\mathrm{H}-\mathrm{D}$ configuration). The higher rotational states of $\mathrm{HD}(J>2)$ preferentially form $\mathrm{XD}^{+}$ at all collision energies.

\section{Summation over rotational states}

Because the HD molecules will be distributed over many rotational states, the fraction of all collisions in which $\theta$ is between $-\pi / 2$ and $\pi / 2$ is

$$
R(E)=\sum_{J=0}^{\infty} P(J) R(J, E),
$$

where $R(J, E)$ is given by Eq. (23) and where $P(J)$, the probability of finding an HD molecule in the $J$ th vibrational state, is given by

$$
P(J)=(2 J+1)(B / k T) \exp [-J(J+1) B / k T] .
$$

$T$ is the temperature of the target gas ( $358 \mathrm{~K}$ under the

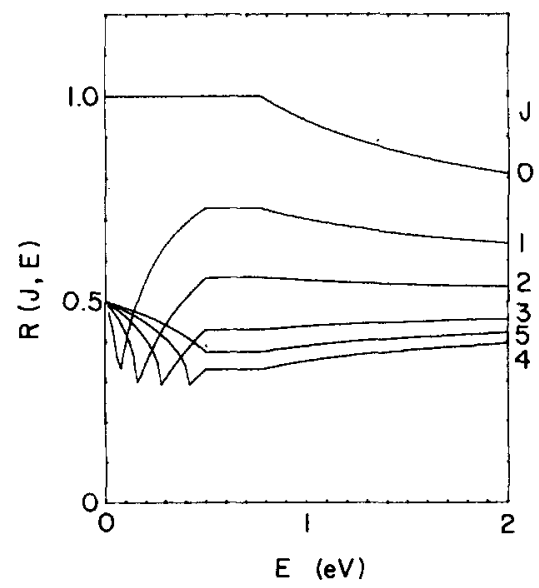

FIG. 3. Fraction of the HD molecules in a given rotational level $J$ that are oriented with their $\mathrm{H}$ end towards the ion $\mathrm{X}^{+}, R(J, E)$, as a function of the relative collision energy $E, R(J, E)$ was calculated from Eq. (23), for the $\mathrm{Kr}^{+}+\mathrm{HD}$ reaction. 
TABLE I. Molecular constants.

\begin{tabular}{lcc}
\hline \hline & $\mathrm{HD}$ & $\mathrm{HD}^{+}$ \\
\hline Vibrational constant, $\omega_{e}\left(\mathrm{~cm}^{-1}\right)$ & $3817^{\mathrm{a}}$ & $1989^{\mathrm{b}}$ \\
Vibrational constant, $\omega_{e} \chi_{e}\left(\mathrm{~cm}^{-1}\right)$ & $95.96^{\mathrm{a}}$ & $49.93^{\mathrm{b}}$ \\
Rotational constant, $B_{e}\left(\mathrm{~cm}^{-1}\right)$ & $45.65^{\mathrm{a}}$ & $22.7^{\mathrm{b}}$ \\
Rotational constant, $\alpha_{e}\left(\mathrm{~cm}^{-1}\right)$ & $1.993^{\mathrm{a}}$ & $0.93^{\mathrm{b}}$ \\
\hline \hline
\end{tabular}

${ }^{2}$ Reference 21.

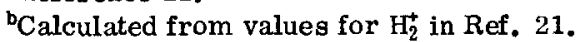

conditions of these experiments), $k$ is the Boltzmann constant, and $B$ is the rotational constant of HD. Since $R(E)$ represents the fraction of collisions leading to $\mathrm{XH}^{+}$ formation, the KIE (expressed as the ratio of the isotopic products) is given by

$$
\frac{\mathrm{XH}^{+}}{\mathrm{XD}^{+}}=\frac{R(E)}{1-R(E)} \text {. }
$$

\section{REACTIONS OF Ar AND Kr ${ }^{+}$WITH HD}

The polarizabilities of $\mathrm{Ar}$ and $\mathrm{Kr}$ were taken as 1.64 $\AA^{3}$ and $2.48 \AA^{3}$, respectively. ${ }^{19}$ The polarizability of HD was assumed to equal that of $\mathrm{H}_{2}\left(0.819 \AA^{3}\right){ }^{20}$ Their hard-sphere radii $r_{e}$ were taken as $r\left(\mathrm{X}^{+}-\mathrm{H}\right)+0.5 r(\mathrm{H}-\mathrm{H})$, where $r(\mathrm{H}-\mathrm{H})$ is $0.74 \AA$ and $r\left(\mathrm{X}^{+}-\mathrm{H}\right)$ was assumed to equal the equilibrium internuclear distance of the isoelectronic hydrogen halide. ${ }^{21}$ Thus, $r_{8}=1.64 \AA$ and 1.78 $\AA$ for the $\mathrm{Ar}^{+}-\mathrm{H}_{2}$ and the $\mathrm{Kr}^{*}-\mathrm{H}_{2}$ systems, respectively. (For these reactions, however, the model is independent of the values chosen for $r_{e}$ because the reactions proceed via a crossing to an $\mathrm{X}-\mathrm{HD}^{+}$surface at distances $r_{x}$ greater than $r_{e}$, so that $r_{x}$ and not $r_{e}$ determines the limiting, high energy value of $r_{0}$ and hence of $\Delta V_{0}$ )

As discussed above and in more detail elsewhere, ${ }^{15}$ the reactions of many ions (including $\mathrm{Ar}^{+}$and $\mathrm{Kr}^{+}$) with hydrogen are known to occur by an "electron jump" to the $\mathrm{X}-\mathrm{H}_{2}^{+}$state during the approach of the reactants. This transition may, in general, be represented by the process

$$
\mathrm{X}^{+}+\mathrm{HD}(v, J)-\mathrm{X}+\operatorname{HD}^{+}\left(v^{\prime}, J^{\prime}\right) .
$$

The energy defects $\Delta \epsilon$ for the various processes represented by Eq. (31) were calculated using the ionization potentials given in Ref. 22 and the molecular constants listed in Table I. The positions of the avoided crossings $r_{x}$ were then calculated from Eq. (11) using the model proposed by Gislason ${ }^{17}$ : the HD target gas was assumed to be in thermal equilibrium and the initial populations $(v, J)$ were calculated from the gas temperature, $J^{\prime}$ was assumed to equal $J$, and $v^{\prime}$ was determined by the assumption that only the outermost crossing need be considered. The values of $r_{x}$ so obtained are listed in Table II.

Kuntz and Roach, ${ }^{15}$ using the diatomics-in-molecules technique, have calculated the lowest potential energy surface for the $\mathrm{Ar}^{+}-\mathrm{H}_{2}$ system. They found that the $\mathrm{Ar}^{+}-\mathrm{H}_{2}$ and $\mathrm{Ar}-\mathrm{H}_{2}^{+}$surfaces cross in the entrance valley when the reactants are about $4 \AA$ apart, a value somewhat greater than those obtained with the simple model used here (see Table II). They also obtained approximate collinear surfaces for $\mathrm{KrH}_{2}^{+}$and found that the crossing in this system was located at a considerably smaller reactant separation. Thus, the values of $r_{x}$ calculated from Eq. (11) are qualitatively correct, although perhaps slightly smaller than the actual values. (Note for the $\mathrm{Kr}^{+}-\mathrm{HD}$ system, several of the $r_{x}$ values are predicted to be smaller than $r_{e}$ )

The kinetic isotope effect, expressed as $\mathrm{XH}^{+} / \mathrm{XD}^{+}$, was calculated as a function of collision energy from Eq. (30), using for $\alpha(\mathrm{HD})$ and $r_{e}$ the values discussed above and for $r_{x}$ the values listed in Table II. The results were weighted to account for the fact that the $\mathrm{X}^{+}$ions are produced by impact of $70 \mathrm{eV}$ electrons and are, therefore, presumably a $2: 1$ mixture of the ${ }^{2} P_{3 / 2}$ and ${ }^{2} P_{1 / 2}$ states. (This weighting took account of the fact that the different states will differ in their $r_{x}$ values, but it was assumed that the two states do not differ in their total cross section for reaction.)

The predicted KIE for the $\mathrm{Ar}^{+}+$HD reaction is compared with the experimental results of Berta et al. ${ }^{4}$ in Fig. 4. The model, which predicts a rather small KIE for this reaction, is in excellent agreement with the experimental data, except at the lowest collision energy $(0.35 \mathrm{eV})$. The fact that the model predicts a somewhat greater KIE than observed at low energy may be an indication that the attractive force in this system is not adequately represented by the ion-induced dipole potential. Because the ionization potentials of $\mathrm{Ar}$ and HD are nearly equal, the true potential may be longer range than the

TABLE II. Energy defects and positions for the transitions $\mathbf{X}^{+}+\mathrm{HD}(v=O, J) \rightarrow \mathbf{X}+\mathrm{HD}^{+}$

\begin{tabular}{|c|c|c|c|c|c|c|c|c|c|}
\hline \multirow[b]{2}{*}{$J$} & \multirow[b]{2}{*}{$P(n)^{\mathrm{a}}$} & \multicolumn{2}{|c|}{$\begin{array}{l}\mathrm{X}^{+}=\mathrm{Ar}^{+}\left({ }^{2} P_{3 / 2}\right) \\
v^{\prime}=2\end{array}$} & \multicolumn{2}{|c|}{$\begin{array}{l}\mathrm{X}^{+}=\mathrm{Ar}^{+}\left({ }^{2} P_{1 / 2}\right) \\
v^{\prime}=3\end{array}$} & \multicolumn{2}{|c|}{$\begin{array}{l}\mathrm{X}^{+}=\mathrm{Kr}^{+}\left({ }^{2} P_{3 / 2}\right) \\
v^{\prime}=0\end{array}$} & \multicolumn{2}{|c|}{$\begin{array}{l}\mathrm{X}^{+}=\mathrm{Kr}^{+}\left({ }^{2} P_{1 / 2}\right) \\
v^{\prime}=0\end{array}$} \\
\hline & & $\Delta \in(\mathrm{eV})$ & $r_{x}(\AA)$ & $\Delta \epsilon(\mathrm{eV})$ & $r_{x}(\AA)$ & $\Delta \epsilon(\mathrm{eV})$ & $r_{x}(\AA)$ & $\Delta \epsilon(\mathrm{eV})$ & $r_{x}(\AA)$ \\
\hline 0 & 0.180 & 0.157 & 2.48 & 0.188 & 2.37 & 1.461 & 1.69 & 0.855 & 1.93 \\
\hline 1 & 0.376 & 0.151 & 2.50 & 0.182 & 2.39 & 1.456 & 1.69 & 0.850 & 1.94 \\
\hline 2 & 0.306 & 0.139 & 2.55 & 0.169 & 2.43 & 1.445 & 1.70 & 0.839 & 1.94 \\
\hline 3 & 0.145 & 0.121 & 2.64 & 0.151 & 2.50 & 1.428 & 1.70 & 0.822 & 1.95 \\
\hline 4 & 0.044 & 0.097 & 2.79 & 0.125 & 2.62 & 1.405 & 1.71 & 0.799 & 1.97 \\
\hline 5 & 0.009 & 0.067 & 3.06 & 0.094 & 2.82 & 1.378 & 1.72 & 0.772 & 1.98 \\
\hline 6 & 0.001 & 0.030 & 3.75 & 0.056 & 3.20 & 1.344 & 1.73 & 0.738 & 2.00 \\
\hline
\end{tabular}
$\left(v^{\prime}, J\right)$

${ }^{2} P(J)$ is the population of the $J$ th rotational level of $\mathrm{HD}$ at $358^{\circ} \mathrm{K}$, as calculated from

Eq. (29). 
$r^{-4}$ potential assumed here. ${ }^{23}$ At low collision energies this would cause the centrifugal barrier to occur at greater distances of separation, $r_{\text {max }}$, thereby decreasing $\Delta V_{0}$ and the predicted KIE.

The predicted KIE for the $\mathrm{Kr}^{+}+\mathrm{HD}$ reaction is compared with available experimental results in Fig. 5 . The agreement at low energy $(E<1 \mathrm{eV})$ is very good, with the model predicting both the steep initial rise and the subsequent plateau in the KIE. The results at higher collision energies are less good, with the model overestimating the magnitude of the KIE. This behavior is presumably due to the rather arbitrary nature of the high energy cutoff function employed in this model and the fact that a different mechanism (which leads to $\mathrm{KrH}^{+} / \mathrm{KrD}^{+}$ratios less than unity) is becoming operative at these higher collision energies. A possible cause for this high energy, inverse KIE has been discussed in the preceding paper. ${ }^{2}$ The model also slightly overestimates the magnitude of the KIE at its maximum. There are two possible causes for this. First, the thermal motion of the HD target molecules gives rise to a spread in collision energies ( $F W H M=0.48 \mathrm{eV}$ at $E=0.7 \mathrm{eV}$ ), ${ }^{24}$ thus smearing out the KIE and decreasing its apparent magnitude. Second, the true $r_{x}$ values are probably somewhat greater than the values calculated from Eq. (11); use of larger $r_{x}$ values would result in a somewhat smaller predicted KIE at collision energies above 0.5 eV.

\section{SUMMARY}

An orientation isotope effect is proposed which is based upon (1) the fact that the displacement of the center of polarizability from the center of mass in the HD molecule will cause the intermolecular potential to contain a term of the form $\Delta V(r) \cos \theta$; (2) the fact that, for many ions $\mathrm{X}^{+}$, reaction occurs by a crossing from the initial $\mathrm{X}^{+}-\mathrm{HD}$ surface to an $\mathrm{X}-\mathrm{HD}^{+}$surface; (3) the assumption that the ratio $\mathrm{XH}^{+} /\left(\mathrm{XH}^{+}+\mathrm{XD}^{+}\right)$is equal to the fraction of $\mathrm{HD}$ molecules with their $\mathrm{H}$ end oriented toward the ion $\mathrm{X}^{+}$at the moment the reactants pass over the centrifugal barrier in the effective radial potential (or cross over to the $\mathrm{X}-\mathrm{HD}^{+}$surface, if the crossing

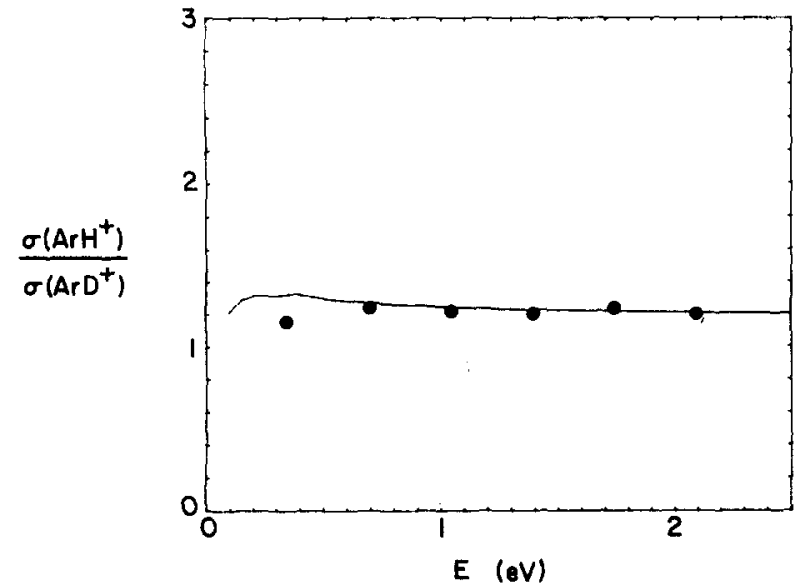

FIG. 4. Comparison of the calculated product ratio, $\mathrm{ArH}^{+} / \mathrm{ArD}^{*}$ (solid line) with experimental results obtained by Berta et al. ${ }^{4}$ (solid circles) vs relative collision energy $E$.

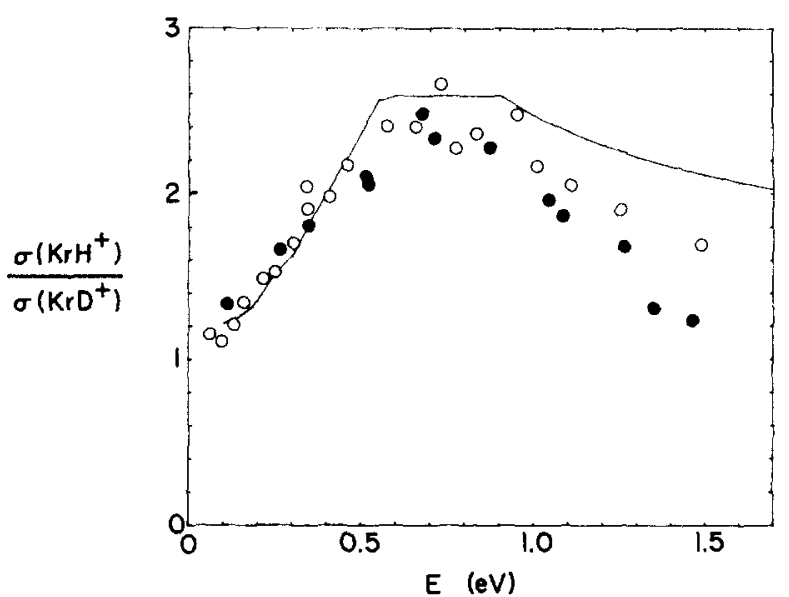

FIG. 5. Comparison of the calculated product ratio, $\mathrm{KrH}^{+} / \mathrm{KrD}^{+}$ (solid line) with experimental results obtained in the present study (solid circles) and by Henchman and co-worker ${ }^{1}$ (open circles) vs relative collision energy $E$.

occurs prior to the attainment of the centrifugal barrier). Expressions are derived which permit the calculation of the KIE once an analytic expression has been assumed for the intermolecular potential.

Qualitatively, three effects can be discerned: (1) at low collision energies $\left(\Delta V<E_{J}\right)$, the HD rotates at an uneven speed, spending the majority of the time with its $\mathrm{D}$ end directed towards the ion and hence favoring $\mathrm{XD}^{+}$ formation; (2) at moderate collision energies $\left(\Delta V>E_{J}\right)$, the rotation of HD is hindered, with the $\mathrm{H}$ end becoming locked in towards the ion and thereby favoring $\mathrm{XH}^{+}$formation; (3) at very high energies collision occurs on a time scale short compared to a rotational period and the HD orientation upon collision is essentially random, resulting in equal production of $\mathrm{XH}^{+}$and $\mathrm{XD}^{+}$.

Assumption of the ion-induced dipole potential has permitted quantitative application of this model to the reactions of $\mathrm{Ar}^{+}$and $\mathrm{Kr}^{+}$with $\mathrm{HD}$. As shown in Figs. 4 and 5 , the agreement between experiment and this model is very good: the model both predicts the large KIE in the $\mathrm{Kr}^{+}$reaction at collision energies less than $1.5 \mathrm{eV}$ (c. m. ) and accounts for the near absence of such an effect in the $\mathrm{Ar}^{+}$reaction. In view of the assumptions made in the formulation of this model, the close agreement with the experimental data is perhaps somewhat fortuitous. Nevertheless, the fact that the model is physically plausible and, without the use of adjustable parameters, quantitatively reproduces the KIE's observed for these two ion-molecule reactions, supports the hypothesis that the orientation effect described here is a major factor producing the unusual KIE observed in the $\mathrm{Kr}^{+}+\mathrm{HD}$ reaction at low collision energies.

The remarkable difference in the KIE's displayed by $\mathrm{Ar}^{+}$and $\mathrm{Kr}^{+}$apparently arises from the difference in the positions of the crossings of the $\mathrm{X}^{+}-\mathrm{HD}$ and $\mathrm{X}-\mathrm{HD}^{+}$potential energy surfaces. Because the ionization potential of $\mathrm{X}$ is the major factor in determining the position of the crossing, one might expect similar KIE's in the reactions of $\mathrm{Ar}^{+}$and $\mathrm{N}_{2}^{+}$with $\mathrm{HD}$, for example, as a consequence of the virtual equality of the ionization poten- 
tials of $\mathrm{Ar}$ and $\mathrm{N}_{2}$.

Despite its apparent success, however, the model's limitations must be recognized. First, the results are sensitive to the intermolecular potential, the exact form of which will be unknown in most cases. Although the ion-induced dipole potential appears (perhaps fortuitously) to be satisfactory in the reactions considered here, it may not be adequate to describe other reaction systems. One obvious improvement to the simple model proposed here, therefore, would be the use of a more realistic intermolecular potential. Second, consideration must be given to other possible causes of kinetic isotope effects, including quantum effects (such as densities of states) and kinematic restraints arising from energy and momentum conservation. Third, Eq. (16), although allowing the essential features of the model to be discerned more readily, should be replaced by an expression which properly averages over the initial orientations of the HD rotational angular momentum.

\section{ACKNOWLEDGMENTS}

Acknowledgment is made to the donors of the Petroleum Research Fund, administered by the American Chemical Society, for partial support of this research. Additional support was provided by the Research Corporation and by the University of Kansas General Research Fund. The author would like to express his thanks to Professor M. J. Henchman for calling this problem to his attention and for helpful discussions on the subject.

${ }^{1} \mathrm{P}$. Fennelly, Ph. D. thesis, Brandeis University, 1972; P. Fennelly, M. J. Henchman, and A. S. Werner (unpublished results).
${ }^{2}$ S. Chivalak and P. M. Hierl, J. Chem. Phys. 67, 4654 (1977), preceding article.

${ }^{3}$ F. S. Klein and L. Friedman, J. Chem. Phys. 41, 1789 (1964).

${ }^{4}$ M. A. Berta, B. Y. Ellis, and W. S. Koski, Adv. Chem. Ser. 58, 80 (1966).

${ }^{5}$ A. Henglein, K. Lacmann, and B. Knoll, J. Chem. Phys. 43, 1048 (1965); A. Henglein, Adv, Chem. Ser. 58, 63 (1966).

${ }^{6}$ T. F. Moran and L. Friedman, J. Chem. Phys. 42, 2391 (1965).

${ }^{7} \mathrm{~J}$. C. Tully, Ber. Bunsenges. Phys. Chem. 77, 557 (1973).

${ }^{8}$ P. J. Kuntz and A. C. Roach, J. Chem. Phys. 69, 6299 (1973).

${ }^{9}$ K. T. Gillen, B. H. Mahan, and J. S. Winn, J. Chem. Phys. 58, 5373 (1973); ibid. 69, 6380 (1973).

${ }^{10} \mathrm{~J}$. H. Futrell and F. P. Abramson, Adv. Chem. Ser. 58, 107 (1966).

${ }^{11}$ P. M. Hierl, Z. Herman, and R. Wolfgang, J. Chem. Phys. 53, 660 (1970).

${ }^{12}$ R. J. Suplinskas, J. Chem. Phys, 48, 5046 (1968).

${ }^{13}$ J. C. Light and S. Chan, J. Chem. Phys. 51, 1008 (1969).

${ }^{14}$ T. F. George and R. J. Suplinskas, J. Chem. Phys, 51, 3666 (1969); ibid. 54, 1037, 1046 (1971).

${ }^{15}$ P. J. Kuntz and A. C. Roach, J. Chem. Soc. Faraday Trans. 2 68, 259 (1972).

${ }^{16}$ See, for example, M. J. Henchman, Ion-Molecule Reactions, edited by J. L. Franklin (Plenum, New York, 1972), pp. 101259.

${ }^{17}$ E. A. Gislason, J. Chem. Phys. 57, 3396 (1972).

${ }^{18}$ T. Su and M. T. Bowers, J. Chem. Phys. 58, 3027 (1973).

${ }^{19}$ E. W. Rothe and R. B. Bernstein, J. Chem. Phys. 31, 1619 (1959).

${ }^{20}$ N. J. Bridge and A. D. Buckingham, Proc. R. Soc. London Ser. A 295, 334 (1966).

${ }^{21} \mathrm{G}$. Herzberg, Spectra of Diatomic Molecules (Van Nostrand, Princeton, NJ, 1950).

${ }^{22}$ J. L. Franklin, J. G. Dillard, H. M. Rosenstock, J. T. Herron, K. Draxl, and F. H. Field, Natl. Stand. Rep. Data Ser. Natl. Bur. Stand. 26 (1969).

${ }^{23}$ J. C. Light and J. Lin, J. Chem. Phys. 43, 3209 (1965).

${ }^{24}$ P. J. Chantry, J. Chem. Phys. 55, 2746 (1971). 